\title{
LA UTILIDAD DEL ANÁLISIS DEL RELIEVE EN BENEFICIO DE LA SOCIEDAD
}

\author{
The usefulness of the relief analysis for the benefit of society
}

\section{EPISTEMUS}

ISSN: 2007-8196 (electrónico)

ISSN: 2007-4530 (impresa)

Grisel Alejandra Gutiérrez Anguamea ${ }^{1}$ Alba Lucina Martínez Haros² José Ismael Minjárez Sosa ${ }^{3}$

Recibido: 16 de septiembre de 2016, Aceptado: 30 de noviembre de 2016

\section{Autor de Correspondencia:}

M. C. Grisel Alejandra Gutiérrez Anguamea

Correo:grisel@geología.uson.mx

\section{Resumen}

La búsqueda del desarrollo económico y tecnológico de toda organización humana involucra un cambio geográfico local, que se deriva en la implementación de actividades sistemáticas y metodológicas que contribuyan al crecimiento de la sociedad.

El crecimiento de las ciudades y la poca o nula planeación, así como la percepción del ambiente al que pertenecemos, conlleva a una modificación desmedida del entorno y produce el aumento de la vulnerabilidad de la población ante la ocurrencia de desastres de origen natural y antrópico, como los procesos de remoción en masa, inundaciones y escases de agua, entre otros.

En la actualidad, las reglamentaciones gubernamentales para la construcción sugieren la realización de estudios de caracterización de sitio que permiten identificar las condiciones litológicas, estructurales y ambientales [1]; sin embargo, son pocos los reportes que involucran el análisis del relieve como parte del funcionamiento integral del ecosistema inmediato, resultado de la dinámica interna y externa terrestre.

Palabras clave: Vulnerabilidad, ecosistema, relieve.

\section{Abstract}

The economic pursuit and technological development of every human organization involves local geographical change, which is derived in implementing systematic activities and methodologies that contribute to the growth of society.

The growth of cities and the lack of planning and perception of the environment to which we belong, involves an excessive modification of the environment and produces the increased vulnerability of the population to the occurrence of disasters of natural and anthropogenic sources like processes landslides, floods and water shortages, among others.

Currently, government regulations for construction studies suggest characterization of site that identify lithological, structural and environmental conditions [1]; however, few reports involving the analysis of the relief as part of the overall functioning of the ecosystem immediate result of the Earth's internal and external dynamics.

Keywords: Vulnerability, ecosystem, relief. 


\section{INTRODUCCIÓN}

El análisis del relieve consiste en el ordenamiento sistemático y jerárquico de la configuración del terreno, procesos geomorfológicos y fenómenos naturales interrelacionados, producto de los procesos endógenos (estructurales) y exógenos (ambientales) que actúan sobre la superficie terrestre [2].

Los resultados de las investigaciones geomorfológicas son empleados en la evaluación de riesgos y estudios de recursos naturales, debido a la relación entre las propiedades geomórficas del terreno y sus factores ambientales.

Se ha considerado a la geomorfología aplicada como el resultado del análisis geomórfico que lleva hacia el establecimiento de las relaciones ecológicas del paisaje, entre las características de las formas del relieve y los factores ambientales del modelado, como una herramienta en la solución de diversos problemas relacionados con el terreno y de interés práctico para el hombre.

Este tipo de análisis se lleva a cabo obteniendo información que se aplica en un esquema multidisciplinario [3] y su aporte corresponde a la relación entre la expresión del terreno y los agentes, como el clima, el tipo de relieve, la litología, los suelos, la hidrología y la influencia de los organismos vivos, incluyendo el impacto del hombre sobre el paisaje.

\section{DESARROLLO}

Anteriormente, el análisis del relieve era considerado como un apartado complementario en los estudios vinculados a la geografía física; dejando de lado

la contribución de la descripción del paisaje en el entendimiento del ecosistema como una unidad funcional y dinámica del sistema ambiental $[4 ; 5 ; 6]$, producto de la relación entre los componentes bióticos (la vegetación, la fauna, los suelos y los antropismos) y abióticos (el agua, el relieve y el clima).

La dinámica del paisaje está en función de las relaciones internas y externas de dichos componentes, los factores litológicos y morfológicos intervienen en el funcionamiento del componente al que pertenecen $[7 ; 5]$; de tal forma que un sistema de paisajes con sus elementos del relieve comparten un mismo marco climático y morfoestructural, así como una respuesta específica a los procesos de disturbio o regeneración del ambiente $[8 ; 9 ; 5]$.

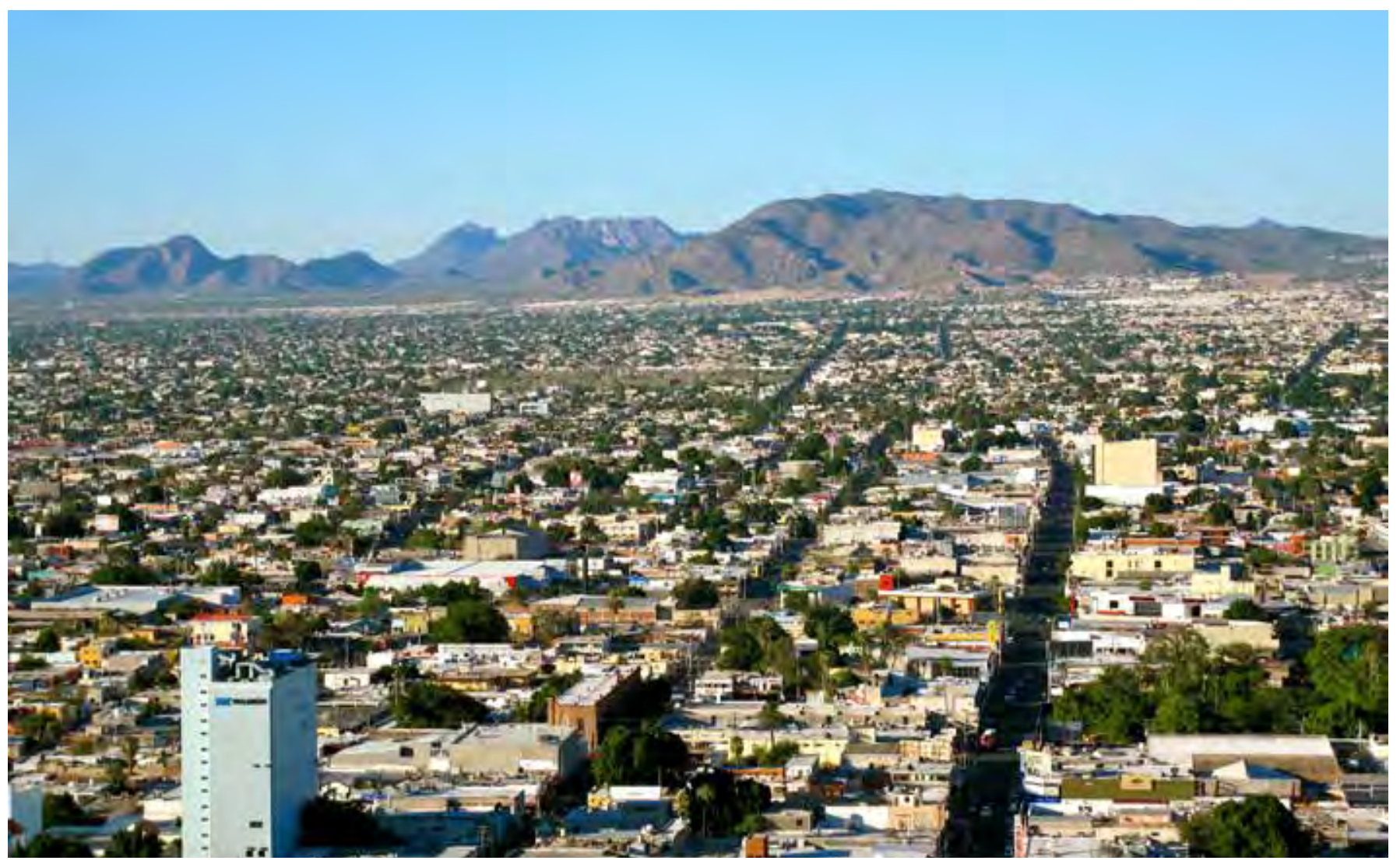




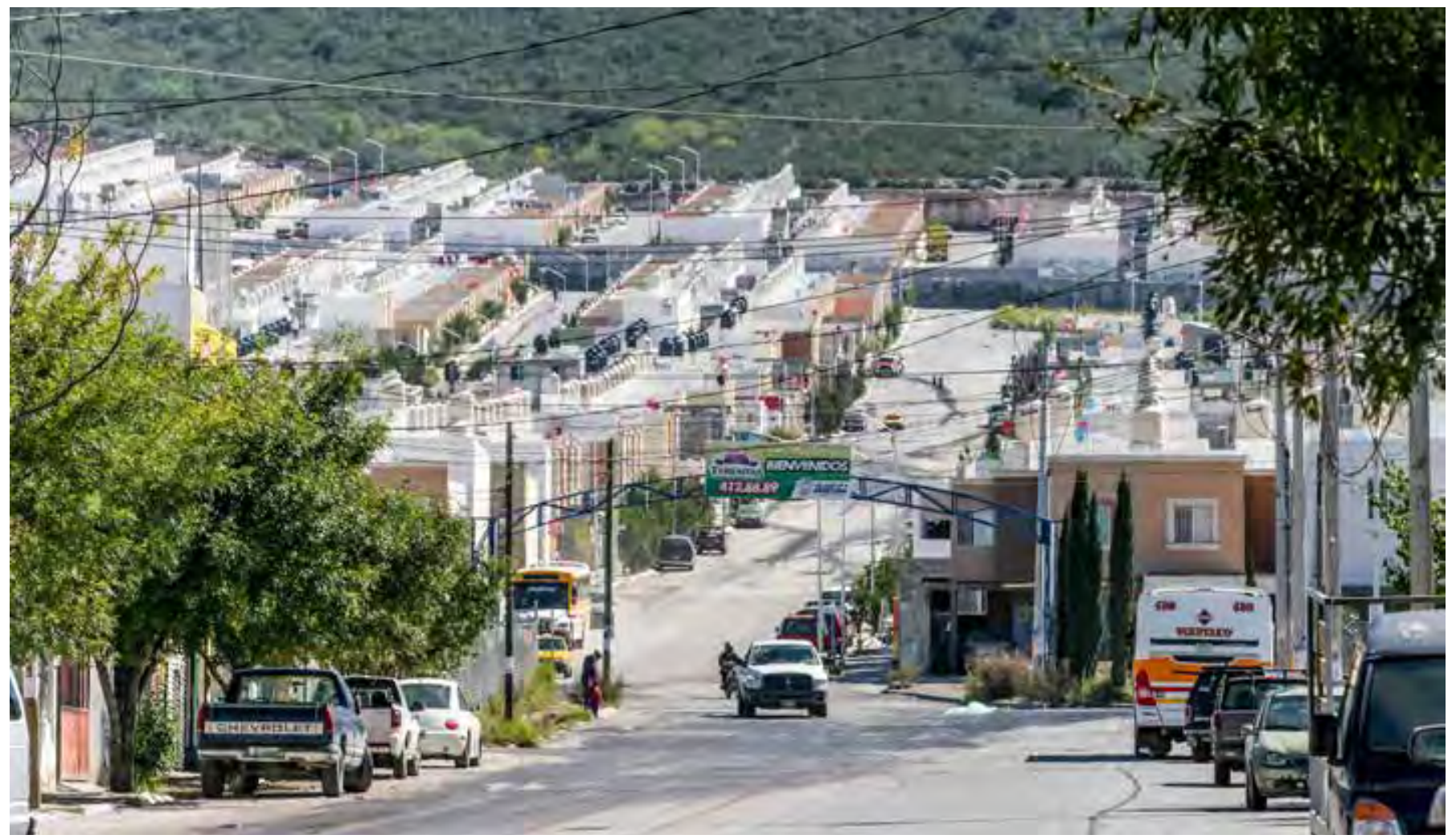

Cuando el nivel de estabilidad de uno de estos componentes es alterado, por ejemplo, el pastoreo extensivo en un rancho, ocasiona modificaciones inducidas antrópicamente en el sitio, provocando una transformación del ecosistema inmediato, esto es al aumentar el número de cabezas de ganado y no considerar el tiempo de recuperación de la vegetación para su alimentación, así como el aumento en la presión superficial en los primeros centímetros del suelo por las pisadas del ganado, desencadenando a una sucesión de eventos que perturban la dinámica de la región.

La consecuente disminución de la cubierta vegetal puede derivar en una pérdida de suelo, debido a que el sustrato se encuentra desprotegido y al estar expuesto a los agentes climáticos (agua, aire, temperatura), aumenta el riesgo de sufrir una erosión potencial del suelo y por ende se incrementan las escorrentías [10]. Por su parte, las escorrentías naturales también se ven afectadas en sus cursos naturales por dicha pérdida de suelo, provocando un cambio en la pendiente general del terreno, ocasionando una variación en la red hidrográfica y a su vez repercute en la eficiencia de la biota endémica. Es decir, un sitio que se mantenía en moderado equilibrio ecológico fue alterado en su funcionamiento por el sobrepastoreo.

En el caso de las restauraciones ecológicas inducidas, es importante destacar el factor tiempo para el área que se pretende restaurar; no basta con realizar grandes actividades de restauración como lo son: obras de acondicionamiento hidrográfico, plantar una gran variedad de especies vegetales e introducir una diversidad animal para que el sistema ecológico local se reactive [11]. El ritmo acelerado de estas acciones le impide a la naturaleza encausarse y reestablecer su equilibrio, por ello la observación del paisaje enfocado en la reestructuración del sitio conlleva una toma de decisiones de forma más consciente.

Cotidianamente, las labores de la sociedad están principalmente enfocadas en el desarrollo económico regional, sin percatarse del impacto que estas producirán a pequeño, mediano y largo plazo en el funcionamiento de su entorno. En cambio, si se analizan las condiciones actuales del relieve como por ejemplo el sistema orográfico en constante movimiento por la acción de los agentes modeladores sobre la superficie terrestre, existe la posibilidad de que los datos obtenidos a través de la observación del medio sean empleados como apoyo en el diagnóstico y mitigación de los problemas que afectan a la sociedad, como los procesos de remoción en masa [12], inundaciones, abatimiento en los niveles freáticos, contaminación y degradación ambiental, incitando al uso racional del ambiente natural o modificado [13], para no alcanzar situaciones de límite que ponen en peligro a las comunidades.

Como se observa en la Figura 1, se presenta la cartografía de las áreas de inundación en la H. de Guaymas, Sonora, por la activación de escorrentías intermitentes que incrementan su descarga en períodos de lluvia y afectan a la población ubicada sobre la planicie de inundación. 


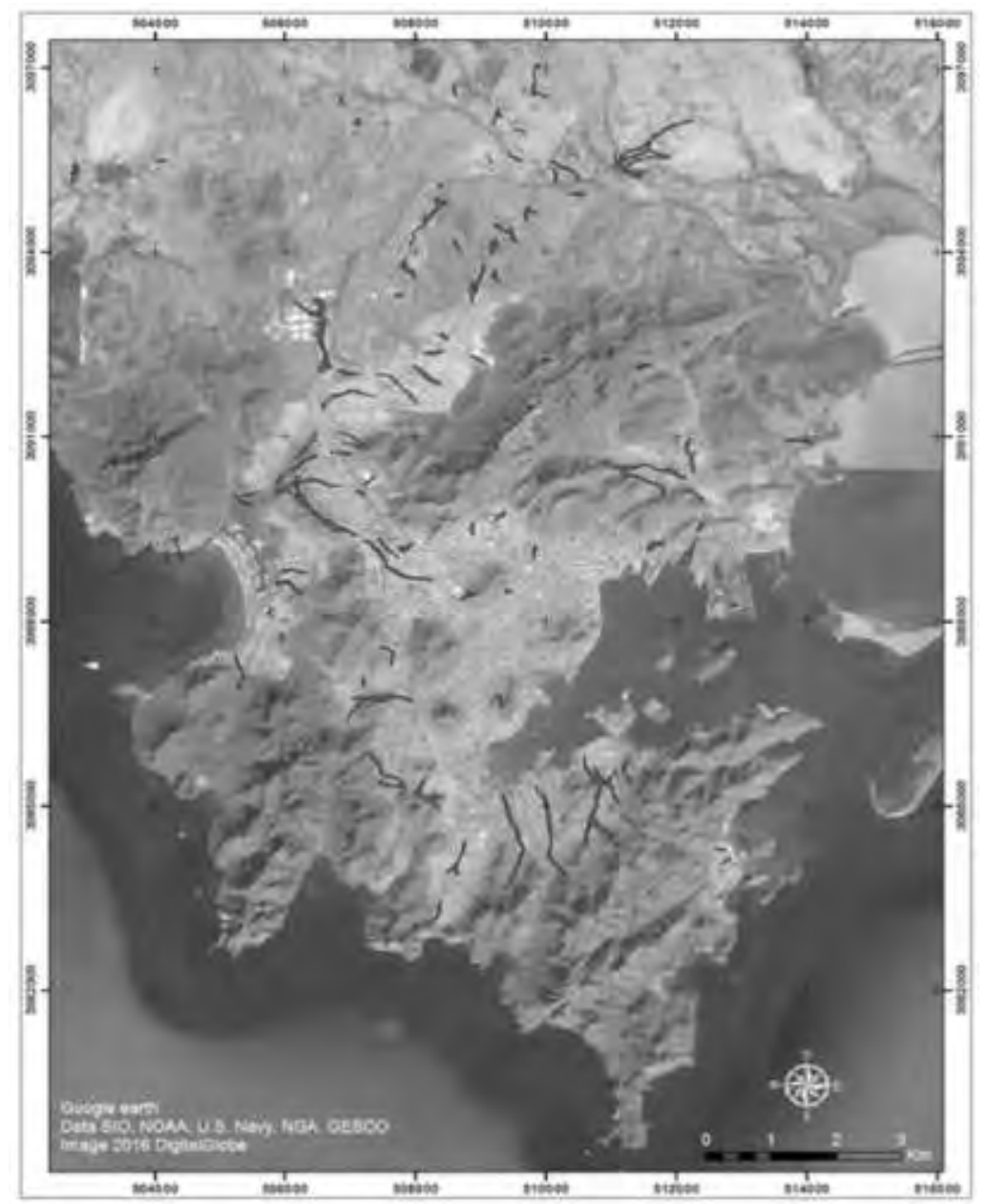

Figura 1. Zonas de inundación en H. Guaymas, Sonora.
El estudio del paisaje proporciona información del arreglo topográfico en función de su aspecto, origen y evolución; involucra conocimiento de los procesos formadores del relieve que ocurren dentro de la corteza terrestre, de los modeladores destructivos y acumulativos del ciclo sedimentario, de la acción de los organismos vivos y de los meteoros que impactan la superficie de la Tierra, que en conjunto moldean el territorio y dan como resultado las geoformas características del lugar.

Una descripción del relieve expone las características naturales y antrópicas del medio, como la cobertura vegetal, las condiciones del suelo, los niveles de erosión, el aumento de áreas de cultivo y urbanizadas, y la concentración de elementos contaminantes. De este modo, permite apreciar las modificaciones ambientales del entorno y es útil en la renovación de cartografías de riesgos, delimitando áreas problemáticas que de manera directa han sido alteradas por el desarrollo de la sociedad.

En la Figura 2 se muestra la cara Sur del Cerro Piedra Bola en la Colonia Pedregal de la Villa, Hermosillo, Sonora. En este lugar se han presentado fenómenos de caída de roca, dichos eventos son detonados por las lluvias y debido a la inclinación de la ladera $\left(20\right.$ a $\left.30^{\circ}\right)$, su inestabilidad incrementa el riesgo de las casas que se ubican en la parte inferior.

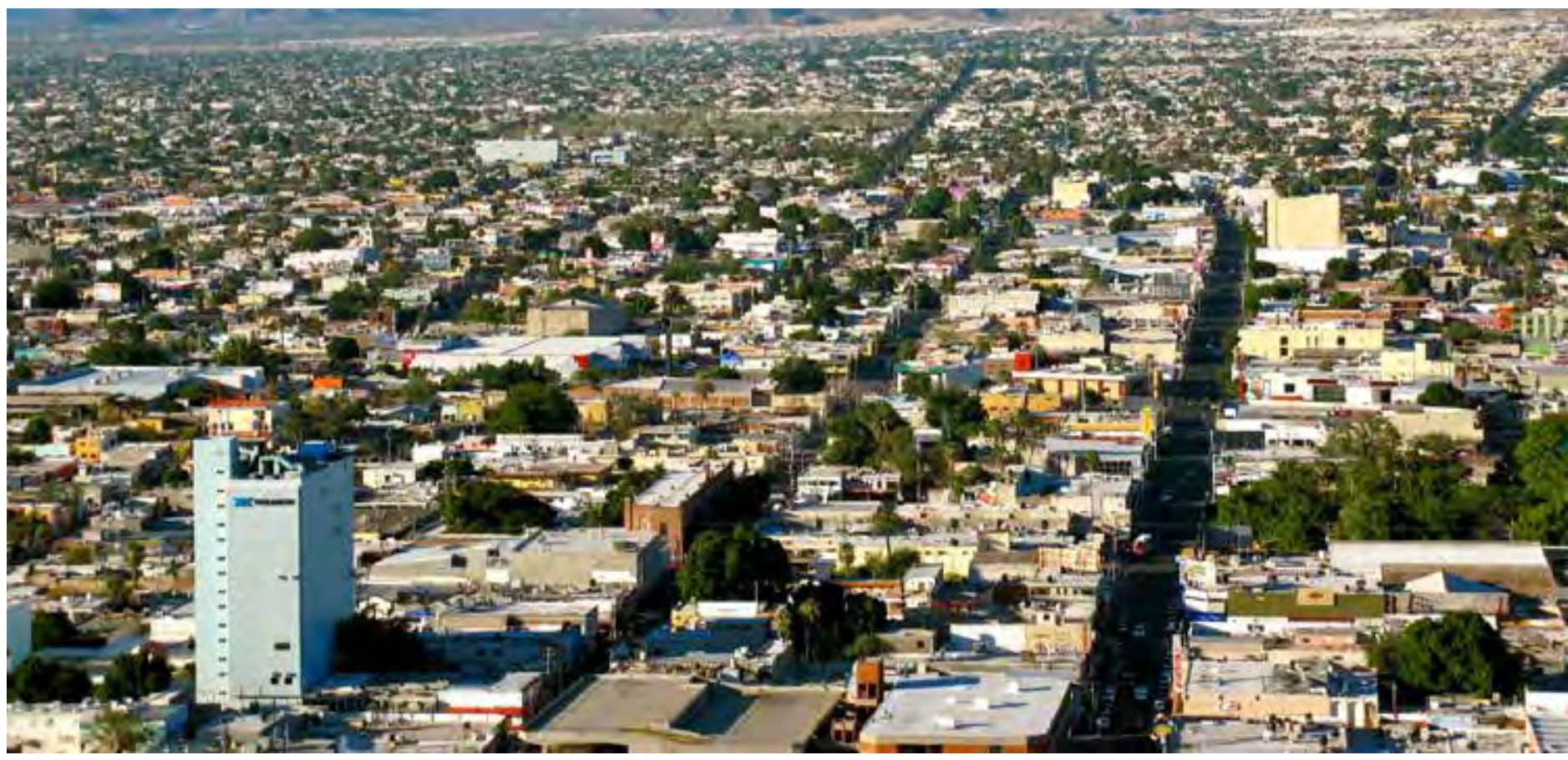




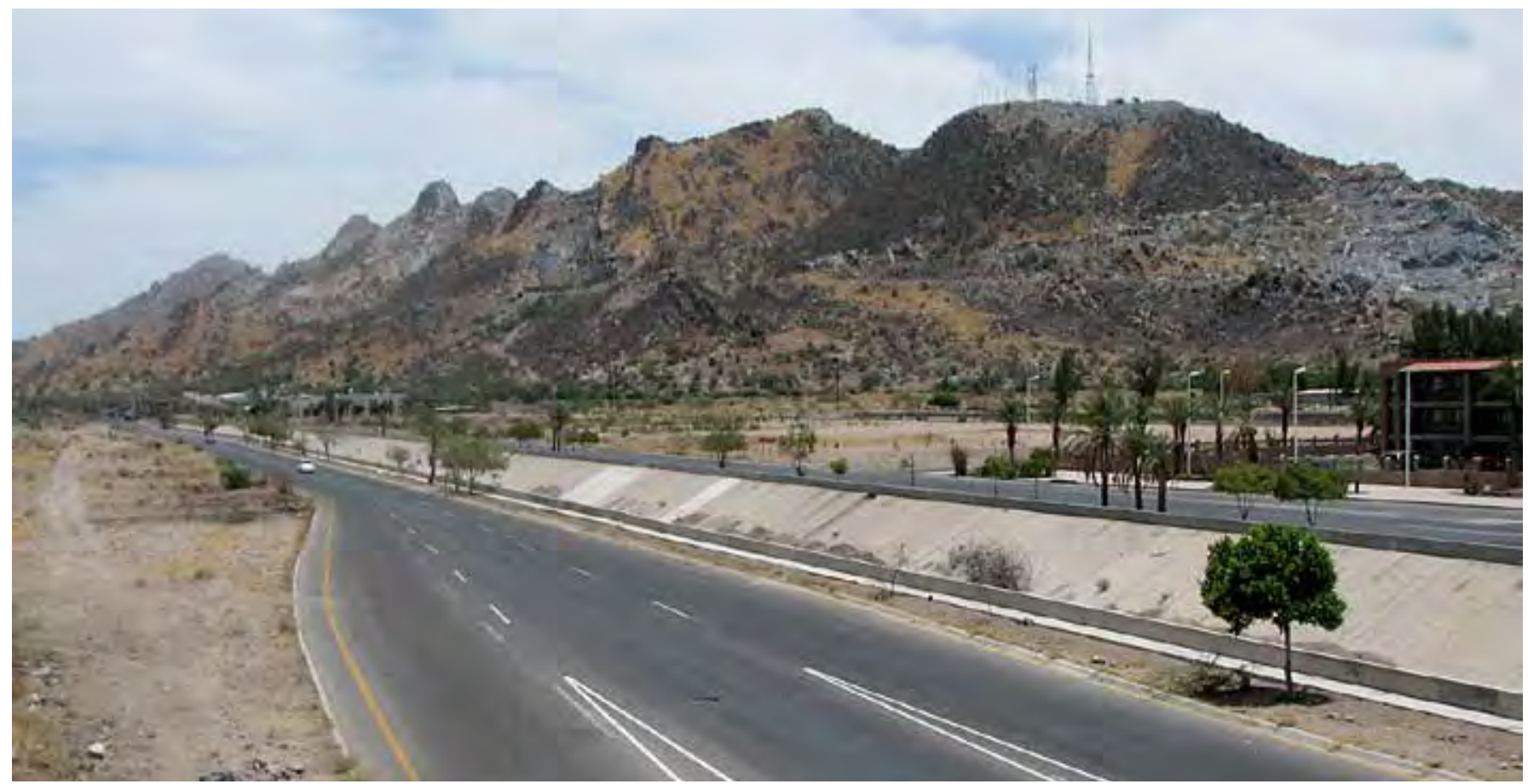

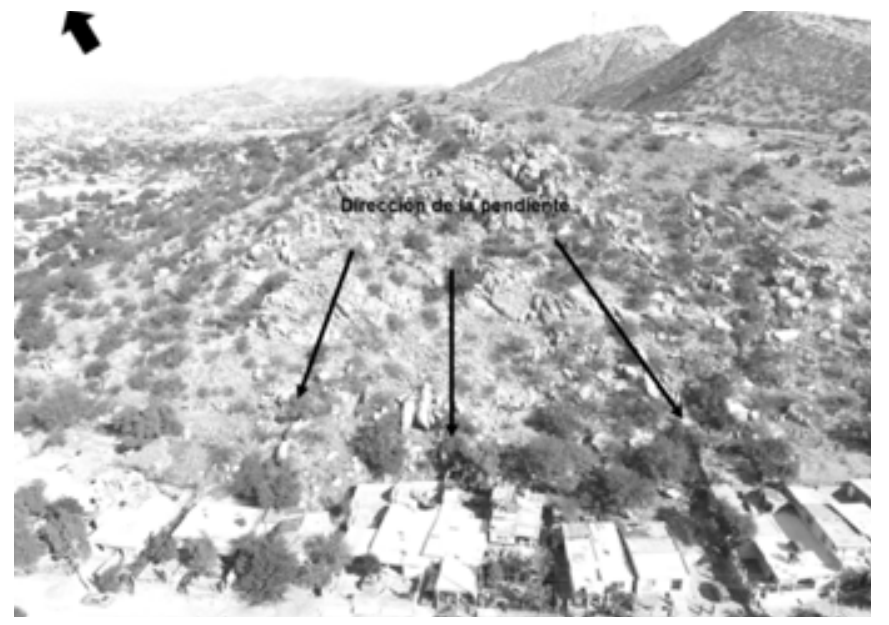

Figura 2. Caída de roca en la Colonia Pedregal de la Villa, Hermosillo, Sonora.

El análisis del relieve permite la exploración de nuevas fuentes de agua subterránea a través de la identificación de zonas favorables para contener el recurso, definidas por la porosidad, permeabilidad y capacidad de almacenamiento del medio para contener agua, producto de su composición litológica, posición topográfica y estructural [14]. La absorción y transmisión de aguas subterráneas no solo depende de las propiedades del subsuelo, también involucra los rasgos geomorfológicos de la superficie; por ejemplo, un relieve granítico de ladera cóncava ubicado en la parte media-alta de una cuenca, no presenta la misma capacidad de retención ni de transmisión del recurso hídrico que un lomerío conglomerático de baja compacidad localizado en la región baja de la misma cuenca. Las condiciones climáticas, topográficas, litológicas $y$ estructurales de ambos son distintas $y$, por ende, su respuesta ante la relación infiltración-escurrimiento también lo será.

Un ejemplo de lo anterior es la Figura 3, donde se clasifica el terreno por su permeabilidad primaria (litología), secundaria (estructura) y geoforma de la región norte de Hermosillo [14].

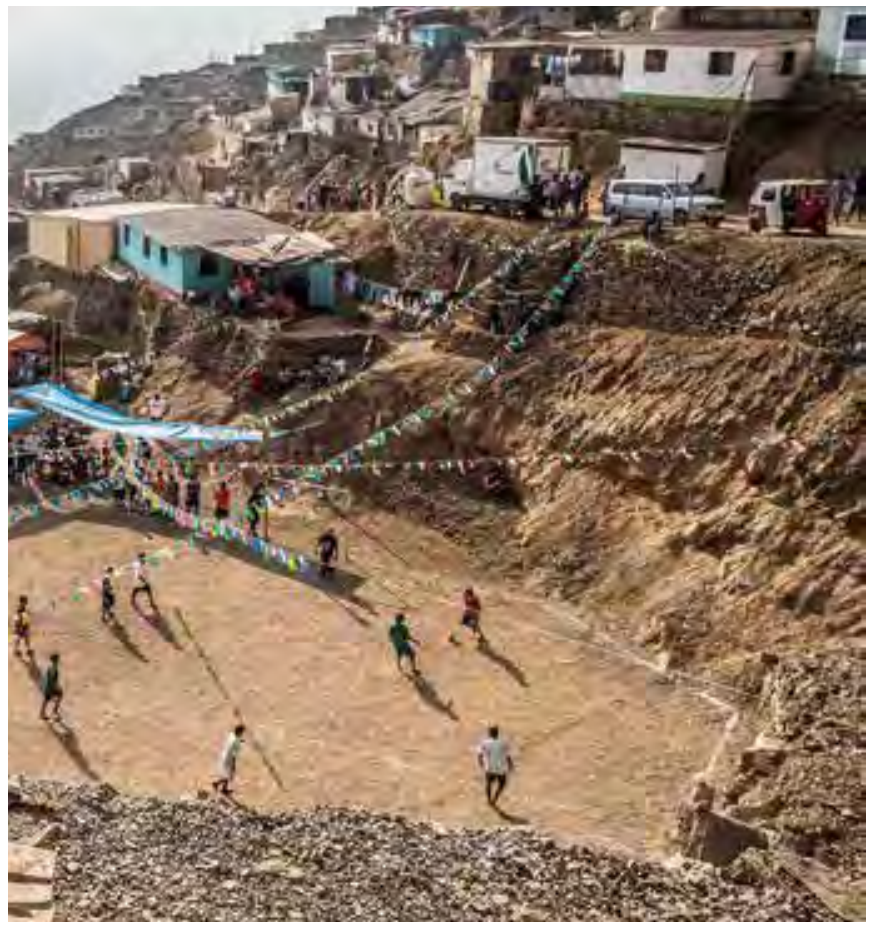




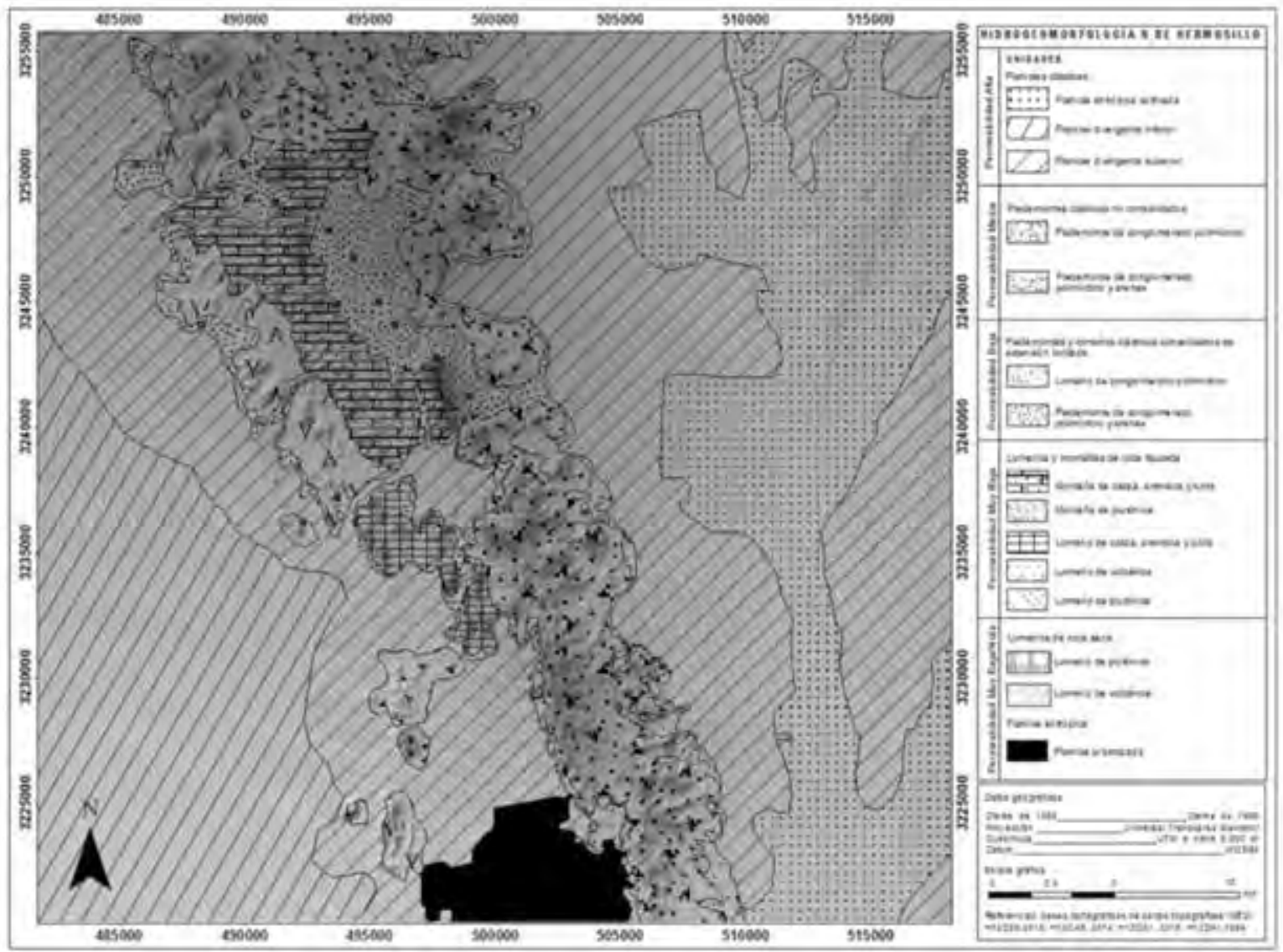

Figura 3. Hidrogeomorfología de la región norte de Hermosillo, Sonora.

El conocimiento adquirido sobre las condiciones del paisaje puede funcionar como una herramienta de apoyo para las dependencias gubernamentales en la toma de decisiones, para evitar la concentración de puntos de extracción de agua subterránea en áreas que presentan un descenso de los niveles piezométricos, posibles problemas de subsidencia, aumento en los costos de explotación, deterioro en la calidad del agua e incluso en el abandono de pozos.

Al controlar las zonas de explotación, se evita que las concesiones se concentren espacialmente en regiones críticas, es decir, se podría relocalizar el punto de extracción hacia zonas acuíferas más favorables, principalmente atendiendo la dirección del flujo subterráneo. De esta manera se podría evitar que en una parte del acuífero sea sobreexplotado y las recomendaciones de relocalización de obras serán más conscientes, permitiendo una reubicación de pozos más viable y un mejor control sobre los volúmenes otorgados.

La observación del terreno permite a la población tener más conocimiento sobre las condiciones en las que se encuentra el estado de salud, su calidad de vida y su patrimonio. Un análisis general del ambiente proporciona información sobre las condiciones que le rodea, de los gases que expiden las zonas industrializadas, de la infiltración de residuos de fertilizantes en tierras de cultivo (planicies cultivadas), del abatimiento del recurso hídrico subterráneo por la intensa concentración de pozos (en sitios posiblemente no óptimos) y su desmedida explotación, de las áreas potencialmente riesgosas

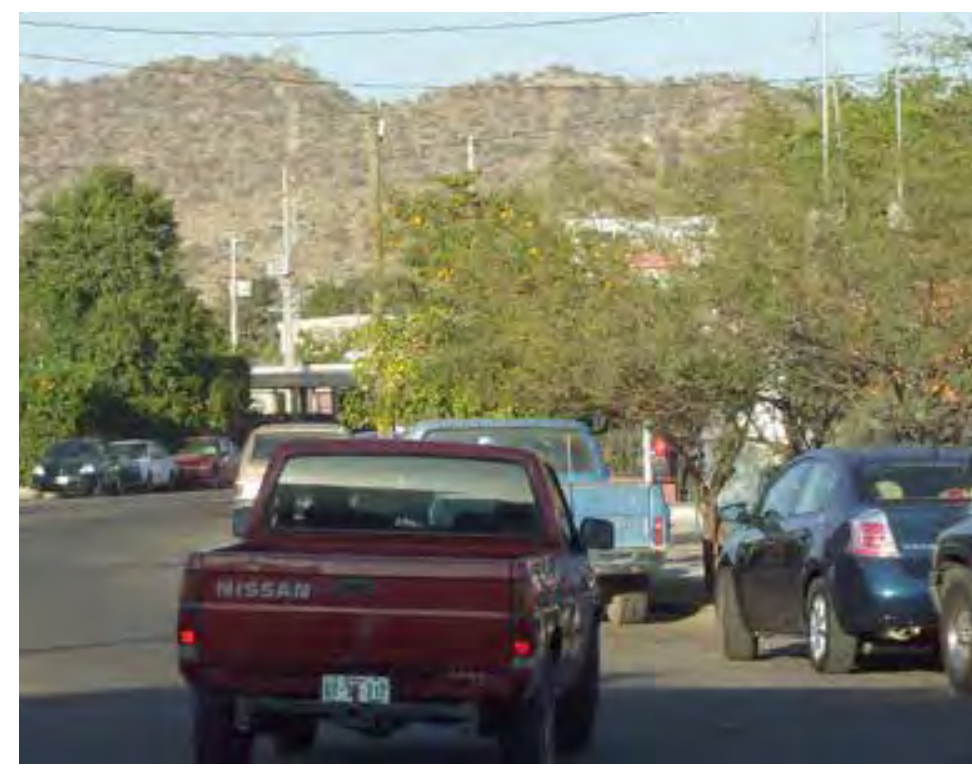


debido a la inestabilidad del terreno y aquellas fácilmente inundables a causa del crecimiento de la urbanización que no toma en consideración el curso hidrográfico natural.

\section{CONCLUSIÓN}

Es importante reconocer las características particulares del terreno debido a que la geomorfología determina las propiedades geológicas, topográficas, estructurales, hidrografías, entre otras del sitio que sientan la base para investigaciones especializadas, en particular para el desarrollo de obras civiles (determinación de áreas idóneas para la construcción de obras hidráulicas como presas, represas, canales y obras de desvío).

El análisis del relieve también puede ser utilizado como apoyo en la elaboración de programas para la protección ambiental, planeación urbana, actividades de reforestación y conservación del suelo, en la delimitación de zonas de riesgo por fenómenos geológicos e hidrometeorológico, como inestabilidad de ladera e inundaciones, y en la caracterización hidrogeológica de un área que presenta situaciones desfavorables en el abastecimiento de agua.

\section{BIBLIOGRAFÍA}

[1] Centro Nacional de Prevención de Desastres, Guía Básica para la Elaboración de Atlas Estatales y Municipales de Peligros y Riesgos, Secretaría de Gobernación, México, D.F., 2006.

[2] M. Panizza, Environmental Geomorphology, Developments in Earth Surface Process 4, The Netherlands, Ed. Elsevier, pp. 1-8, 1996

[3] Th. Verstappen y R. Van Zuidam, The ITC System of Geomorphological Mapping, ITC, Pubication, 10 Enschede, The Netherlands, 1991.
[4] J. Drdos, On the carring capacity of environment. Geografía y Desarrollo, núm. 3/7, pp.19-24, 1992.

[5] A. García-Romero, An evaluation of forest deterioration in the disturbed mountains of western Mexico City. Mountain Research and Development, núm. 22/3, pp. 270-277, 2002.

[6] A. García-Romero y J. Muñoz, El paisaje en el ámbito de la Geografía. México: Instituto de Geografía, UNAM, 2002.

[7 J. Muñoz, Paisaje y Geosistema. Una aproximación desde la Geografía Física. En Martínez de Pisón, E. (ed.). Paisaje y medio ambiente. Soria: Fundación Duques de Soria/Universidad de Valladolid, pp. 45-56, 1998.

[8] R. Forman y M. Godron, Landscape ecology. New York: John Wiley \& Sons, 1986.

[9] T. Gragson, Potential versusactual vegetation: human behavior in a landscape médium. En W. Balée (ed.). Advances in historical ecology, New York: Columbia University, p. 213231, 1998.

[10]J. Ries, M. Seeger y I. Marzolff, Influencia del pastoreo en al cubierta vegetal y la morfodinámica del transecto depresión del Ebro-Pirineos, Departamento de Geografía y Ordenación del territorio, Universidad Zaragoza, núm. 45, pp. 5-19, 2004.

[11]F. Valladares, L. Balaguer, I. Mola, A. Escudero y V. Alfaya, Restauración ecológica deáreas afectadas porinfraestructuras de transporte. Bases científicas para soluciones técnicas. Madrid, España. OFundación Biodiversidad 2011.

[12]A. Martínez, Evaluación de Peligro de Remoción en Masa: Caso Piedra Bola, Hermosillo, Sonora, Tesis de Maestría, Programa Maestría en Ciencias-Geología, Departamento de Geología, Universidad de Sonora, México, 2014.

[13]M. Martínez, Geomorfología ambiental: Universidad Las Palmas de Gran Canaria publ. ISBN 84-89728-16-X, 96 p., 1997.

[14]G. Gutiérrez, Metodología para la elaboración del mapa hidrogeomorfológico de la cuenca del río Asunción, Tesis de Maestría, Programa Maestría en Ciencias-Geología, Departamento de Geología, Universidad de Sonora, México, 2012.

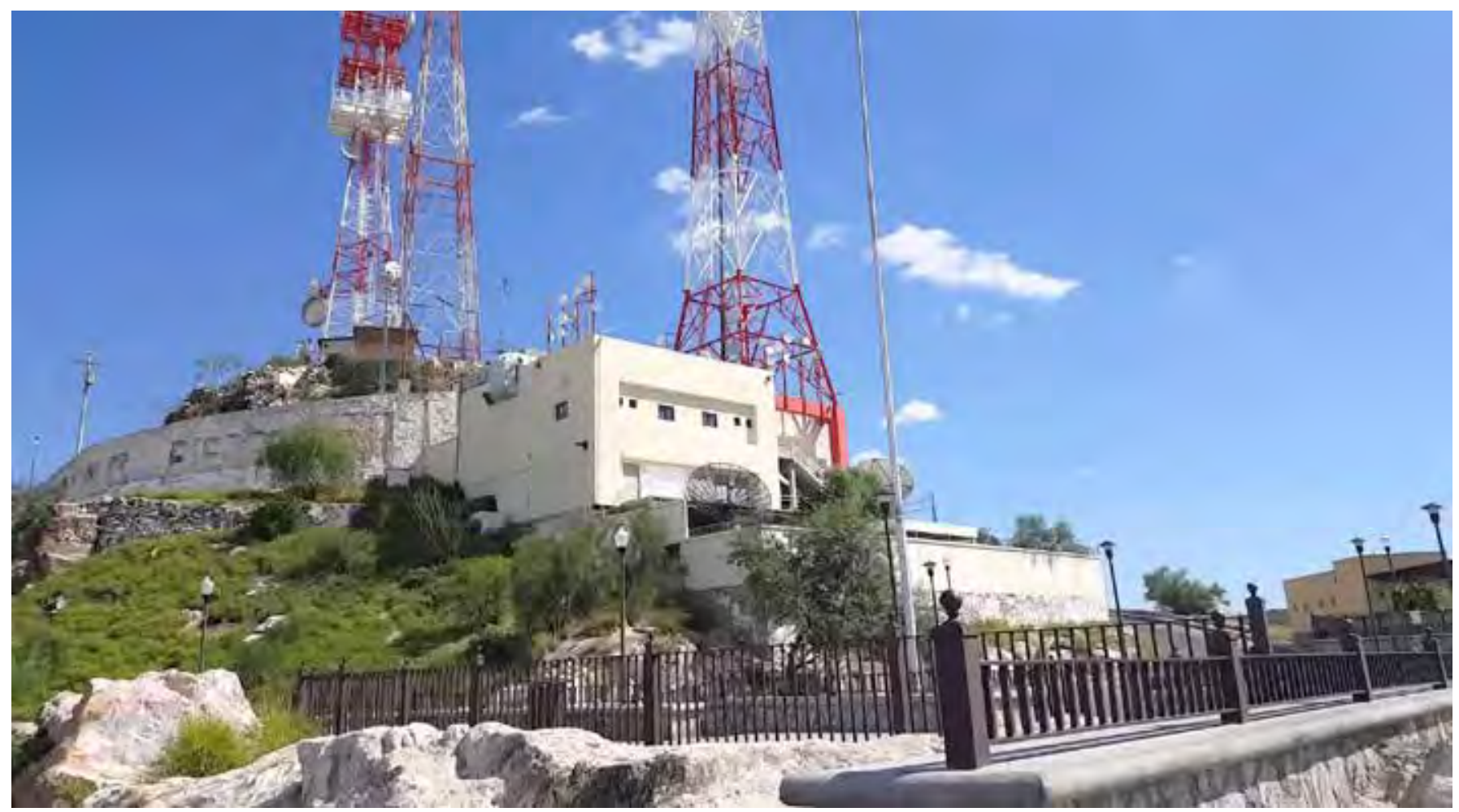

